\title{
THE LAND-USE AND LAND-COVER CHANGE ANALYSIS IN BEIJING HUAIROU IN LAST TEN YEARS
}

\author{
Zhao Qun ${ }^{1}$, Liu Guorong ${ }^{1}$, Tu Jinhua ${ }^{1}$, Wang Zhigang ${ }^{2}$,* \\ 1. Landscape architecture of Beijing University of Agriculture, Beijing, 102206, China.869574897@qq.com \\ 2. China Resources Satellite Application Center, Beijing, 100094, China. Kevinwang2000@163.com
}

\section{Commission III, WG III/7}

KEY WORDS:Land-use and Land-cover,Change Analysis, Remote sensing technology,Beijing Huairou, ECognition

\begin{abstract}
:
With eCognition software, the sample-based object-oriented classification method is used. Remote sensing images in Huairou district of Beijing had been classified using remote sensing images of last ten years. According to the results of image processing, the land use types in Huairou district of Beijing were analyzed in the past ten years, and the changes of land use types in Huairou district were obtained, and the reasons for its occurrence were analyzed.
\end{abstract}

\section{SATELLITE REMOTE SENSING TECHNOLOGY}

\subsection{General Instructions}

Satellite remote sensing technology has the advantages of fast acquisition, wide range and lossless information. It is widely used in land use, resource investigation, environmental testing and disaster assessment. Based on the characteristics of multi-temporal and high resolution of remote sensing data, the role of remote sensing technology in the dynamic monitoring of land use can be fully utilized, which has the characteristic of scientific and accurate, interpretation at any time and smart auxiliary decision-making.

\subsection{Huairou District}

Huairou District is the ecological barriers and important resources guarantee in capital of China. It is a important district in building the integration of urban and rural areas in Beijing. It is also important area of the adjustment and optimization of industrial structure. In this paper, Select Huairou District of Beijing as the research object, as one of the five ecological conservation areas in Beijing. So it's a good study.

\subsection{Object-oriented Method}

Remote sensing technology has developed rapidly and gradually been applied to many fields, as the high resolution satellite launch, how to extract abundant information from high resolution images and satisfy a certain accuracy requirement has become the research

* Corresponding author 
hot spot.

\section{ECOGNITION}

\subsection{Function}

ECognition is a commercially available software for high-resolution telemetry, using the "image object" as a processing unit, using a object-oriented method to categorize data and extract information.

\subsection{Remote sensing image classification}

With the development of Object-oriented technology, the classification algorithm based on object-oriented image segmentation has developed to maturation. The most important characteristic of the classification algorithm is that the smallest unit of classification is the homogeneity Image object (patch), instead of a single pixel, this method can achieve a higher level of remote sensing image classification and extraction of object features. This method is used to segment remote sensing images of Huairou District. Firstly, the homogeneous regions (or elementary unit) are detected and extracted according to the requirements of the remote sensing image classification or the extraction of the target features, (such as spectrum, shape, size, structure, texture, shadow, spatial location, related layout, etc.), so we can achieve the purpose of classification or target feature extraction of remote sensing images.

\subsection{The TM / GF-1 satellite remote sensing data}

With the support of remote sensing images, the TM / GF-1 satellite remote sensing data of the three phases (2006-16, 2016 and 2016) in the last ten years (06-16) were selected as the information source. First of all, preprocessing the remote sensing images, mainly includes image fusion, orthorectification, image registration and image splicing. The next step is image digitization, including vectorization processing, establishing of points, lines, Polygon layers, checking the topology error.

\section{4 obtain the object}

Object-oriented image classification technology through the image multi-scale segmentation to obtain the object, when we classify remote sensing image not only rely on the spectral features of the object corresponding to the target features, but also use its geometric information and structural information, the subsequent image analyzing and processing are also based on the object. And then, combining with the field survey and colour texture analysis, the man computer interactive land classification method was used to classify the land use in Huairou district of Beijing. And after the classification and do some post processing (such as cluster analysis)

\subsection{Land use types distribution map}

Then we obtain the classification results, including the step of choosing a reasonable color scheme, according to the method of visual interpretation, we got the land use types distribution map through the correlation analysis. And do carry on the field research, including selecting the sampling points based on the distribution, designing sampling routes, recording the sampling point at which land use types, and positioning by GPS Finally, do the accuracy verification, including the accuracy of land use types based on field research data, by selecting sample points in Google Earth and loading them to check the accuracy of the classification in ArcGIS 10.2

\section{STRUCTURE AND EVOLUTION FEATURES OF LAND USE IN HUAII DISTRICT FOR NEARLY A DECADE}

\subsection{Advancing Urbanization}

With the support of ArcGIS10.2, by calculating the index of land use pattern, we got the land use structure and evolution characteristics of Huairou district in recent ten years. Urbanization is advancing, and urban sprawl is very obvious to green space erosion. Due to the management system of the construction site, the trend of the land to be replaced by the plain of the plains is more obvious. The total amount of woodland 
is increasing year by year. According to the Beijing policy, every year the government has the task of afforestation. The construction of the city park in Beijing, the planning of the country parks, the reforestation of the farmland, the afforestation of the plains and the afforestation of the mountainous areas have promoted the total amount of forestland. However, we can clearly see that the average distribution of forestland has become a small distribution in the inner suburbs of the city, and the outer suburbs are more dense, and the average degree of forest distribution has decreased significantly, among which, the decrease of the suburbs is obvious, and the urban area has a small increase.

\subsection{Figures}

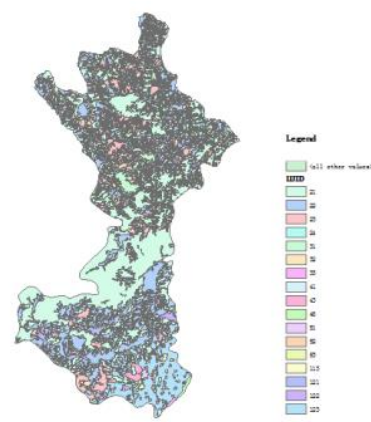

Figure1: Land-use and Land-cover of Huairouin 2000

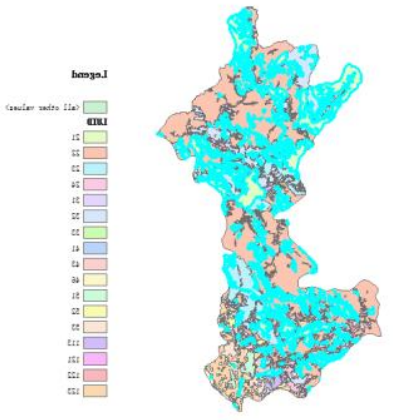

Figure2: Land-use and Land-cover of Huairou in 2006

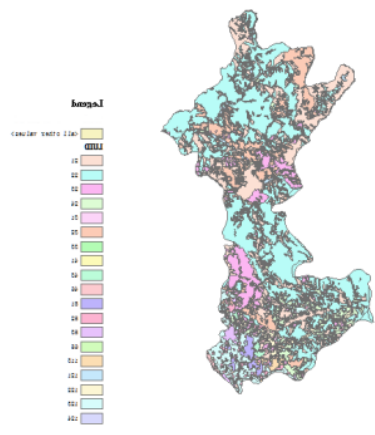

Figure3: Land-use and Land-cover of Huairou in 2012

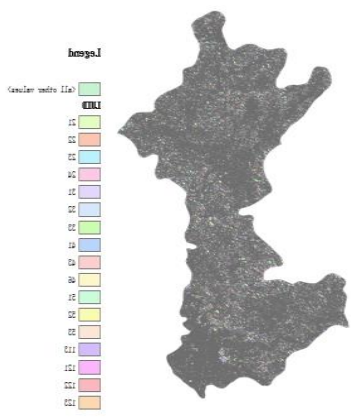

Figure4: Land-use and Land-cover of Huairou in 2016

\section{CONCLUSION}

\subsection{Correlation analysis}

Use a series of related indexes to make a correlation analysis on the temporal and spatial evolution of land use: select four indexes related to land use types from the aspects of nature and social economy, for example: topography, climate, total population and per capita consumption level, Spatial and rasterized processing of the driving factors was carried out, and the spatial overlay analysis was obtained by using ArcGIS10.2 to analyze the spatial overlay analysis of land use, and extract the exponential data to establish the spatial database respectively. Through the analysis of the land use changes in Huairou District, come to a conclusion of the main influencing factors of land use changes in 
recent ten years are economic and social aspects

\section{2 undergone significant changes}

With the economic growth, industrial development and population growth in Beijing, Suburban and rural section has also undergone significant changes in land use, especially in areas close to the built-up area and suburban settlements. Through this study, we can better understand the spatial structure characteristics and dynamic evolution trend of each factor in Huairou District, provide the basis for the urban construction in Huairou District, and provide reference for the ecological barrier construction in Huairou District and provide the technical support of protected zone for the northern Beijing.

\section{REFERENCES}

Huang Jin, 2015a. Study on the classification of high-resolution remote sensing images based on e-cognition.Journal of Heilongjiang Institute of Technology,29(5),pp,16-19.

Lin Tan, Research on Object-Oriented Classification Based on SPOT Images,(1 May,2012).

Chen Yunhao,FengTong,ShiPeijun,Wang-Jinfei,2006a. Based on object oriented and rules.

The remote sensing image is a kind of research. $\mathrm{Wu}$
Han University Journal,31(4),pp,316-318.

FengChao,GuoYunkai,2016a,Object-Oriented-Dynam

ic Analysis of Remote Sensing Vegetation Environment in Remotely Sighted Area, Beijing surveying and mapping,1,pp,39-42.

SunZanyou,QiuBingwen,ChenChongcheng,2009a.Res earch on landscape information extraction based on object-oriented classification technology. Remote sensing application.2,pp,42-46.

ENVI Remote sensing image processing method. Beijing, Science press(Deng Wenbin,2010).

GaoZhiqiang,LiuJiyuan,ZhuangDafang,1999a.Researc $\mathrm{h}$ on the status quo of land use and land cover in China based on remote sensing. Journal of remote sensing,3(2),pp,134-138.

Wang Yan,WangXiaoqing,2009a,AixiaDou,Applicatio $\mathrm{n}$ of Object-Oriented Remote Sensing Classification Method in Seismic Damage Extraction of Wenchuan Earthquake.Earthquake,29(3),pp,54-60.

LiXiaojiang,MengQingyan,WangChunmei,2013a,Obj ect-oriented pixel-level remote sensing image classification method, Journal of Geo-information Science,15(5),pp,744-751. 